\title{
RESEARCH
}

\section{Therapeutic alliance: satisfaction and attrition of patients from a mental health clinic in Ayacucho, Peru}

\author{
Maria C. Prom,, Jeffrey Stovall,, ${ }^{2}$ Luis E. Bedregal,, ${ }^{3}$ James Phillips ${ }^{4}$ \\ and Mario A. Davidson ${ }^{5}$
}

MD Candidate Vanderbilt University School of Medicine, Nashville, Tennessee, USA, email maria.c.prom@vanderbilt.edu

${ }^{2}$ Associate Professor of Psychiatry, Vanderbilt University School of Medicine, Nashville, Tennessee, USA

3 Lecturer in Psychiatry, Yale University School of Medicine,
New Haven, Connecticut, USA ${ }^{4}$ Associate Clinical Professor of Psychiatry, Yale University School of Medicine, New Haven, School of Medicine,
Connecticut, USA

Instructor in Biostatistics, Vanderbilt University School of Medicine, Nashville, Tennessee USA
This study examines the role of the patientprovider relationship (alliance) and patient satisfaction in early patient withdrawal from mental health therapy in rural Peru. A prospective comparison of 60 patients demonstrated that early withdrawal was associated with the clinician's, but not the patient's, evaluation of the patient-provider alliance. This suggests that the satisfaction and alliance questionnaires typically used in high-income countries may not be effective in evaluating patient attitudes in this population, but may be useful for clinician evaluations of the alliance. Clinicians can use the Working Alliance Inventory to indicate the need for early intervention to prevent patient drop-out in middle- and low-income countries.

Early patient withdrawal from mental health therapy is a common problem in middle- and lowincome countries (Lhullier et al, 2000). Patients who have access to mental healthcare often discontinue treatment before improvement of symptoms and quality of life. Patients who leave therapeutic programmes early have poorer outcomes; therefore, it is essential to find methods to reduce early patient withdrawal (Rossi et al, 2008).

Although there are no data for low-income countries, research in high-income countries suggests that $57.6-67.2 \%$ of patients will require a minimum of 12.7 sessions of evidence-based interventions in order to recover (Hansen et al, 2002). Many patients do not fulfil this minimum, as indicated in a recent review, which found that 20-70\% of patients terminate their therapy after the first session, $50 \%$ by the third session and up to $65 \%$ by the tenth session, giving an overall estimated attrition rate of $47 \%$ (Barrett et al, 2008).

Premature patient withdrawal results from a variety of factors, ranging from the qualities of the treatment programme to patient and clinician characteristics. Patient characteristics that affect premature termination include gender, age, income level, minority status, substance misuse, occupational stability, psychiatric diagnosis, expectations regarding therapy and academic achievement. Characteristics of the treating clinician that influence premature termination include patient-provider gender match, expectation of patient improvement, empathy for the patient and skill level. Other factors that affect patient withdrawal include the type of therapy (e.g. pharmacological versus behavioural), strength of the patient-provider relationship, length of delay to first appointment and accessibility of clinics. The majority of these factors are cross-cultural and have been found to be important in multiple studies worldwide (Edlund et al, 2002; Barrett et al, 2008; Morlino et al, 2009; Reneses et al, 2009).

The strength of the patient-provider relationship we here term therapeutic alliance, a concept characterised by how comfortable the patient and treating clinician are with one another and the therapy plan (Martin et al, 2000; Santibáñez Fernández et al, 2009). This factor has not been well studied outside of high-income countries. The present study explores this aspect as a factor in early patient withdrawal from a rural Peruvian mental health clinic.

\section{Method}

\section{Setting and sampling strategy}

The study was conducted at a free mental health out-patient clinic in the rural Andes city of Ayacucho, Peru, which serves a low-income population. The study participants consisted of 60 Spanish-speaking patients, aged 18-66 years, completing their first out-patient consultation with a psychologist or psychiatrist. After giving their informed consent, participants completed the Spanish-language client versions of the Working Alliance Inventory (WAI) and the Satisfaction with Services (SWS) questionnaire following their first out-patient therapy session. To prevent biases in questionnaire responses or behavioural changes in attendance, clinicians did not discuss the study with participants.

The participants' treating clinicians (six psychologists and four psychiatrists) completed the Clinician Questionnaire and the Spanish-language therapist version of the WAI.

Three months following questionnaire completion, the participants' attendance at treatment sessions was evaluated through the clinic's database. Participants who did not attend their second treatment session within 3 months of their first therapy session were categorised as early withdrawers.

Additional data gathered from patient clinical records included age, gender, religion, income, substance misuse history, occupation, diagnosis and education. 


\section{Working Alliance Inventory (WAI)}

The WAI is a 36 -item questionnaire regarding patient and clinician attitudes to their bond and agreement on goals and treatment tasks (Horvath \& Greenberg, 1989). Both the patient and the clinician score each item on a seven-point Likert scale. The Spanish version of the questionnaire (Inventario de Alianza de Trabajo) has been validated by Santibañez (2003).

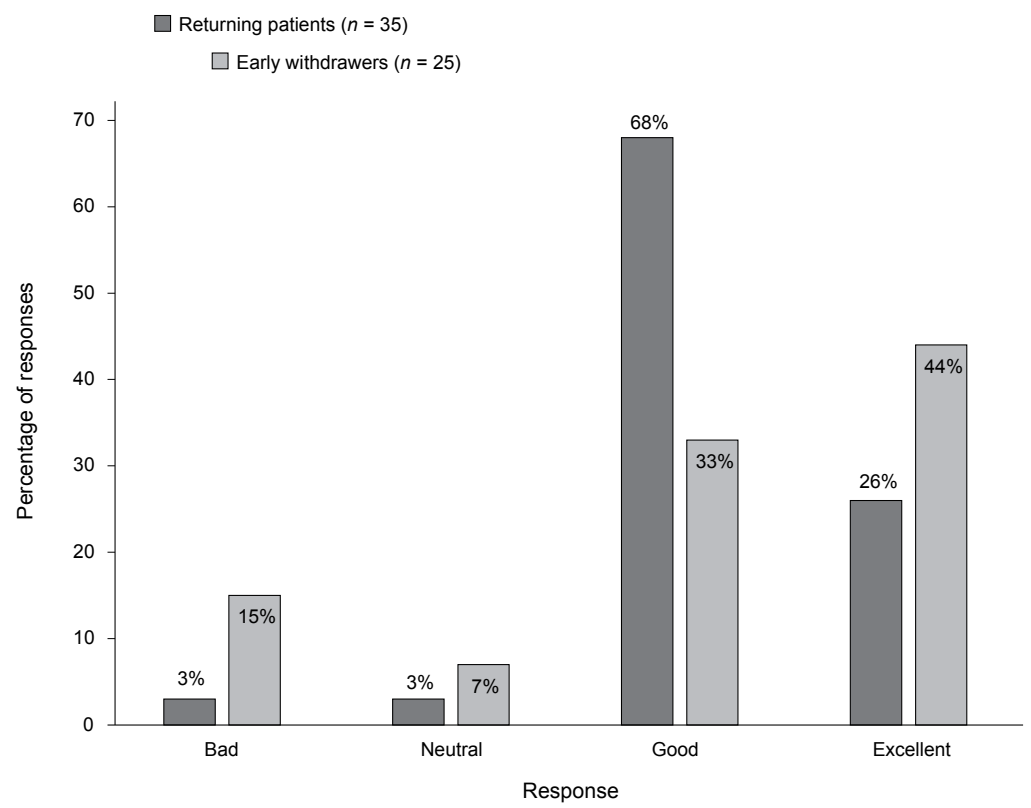

Fig. 1

Patient responses on the Satisfaction with Services (SWS) questionnaire, question 18: 'How would you rate the quality of services you have received?'

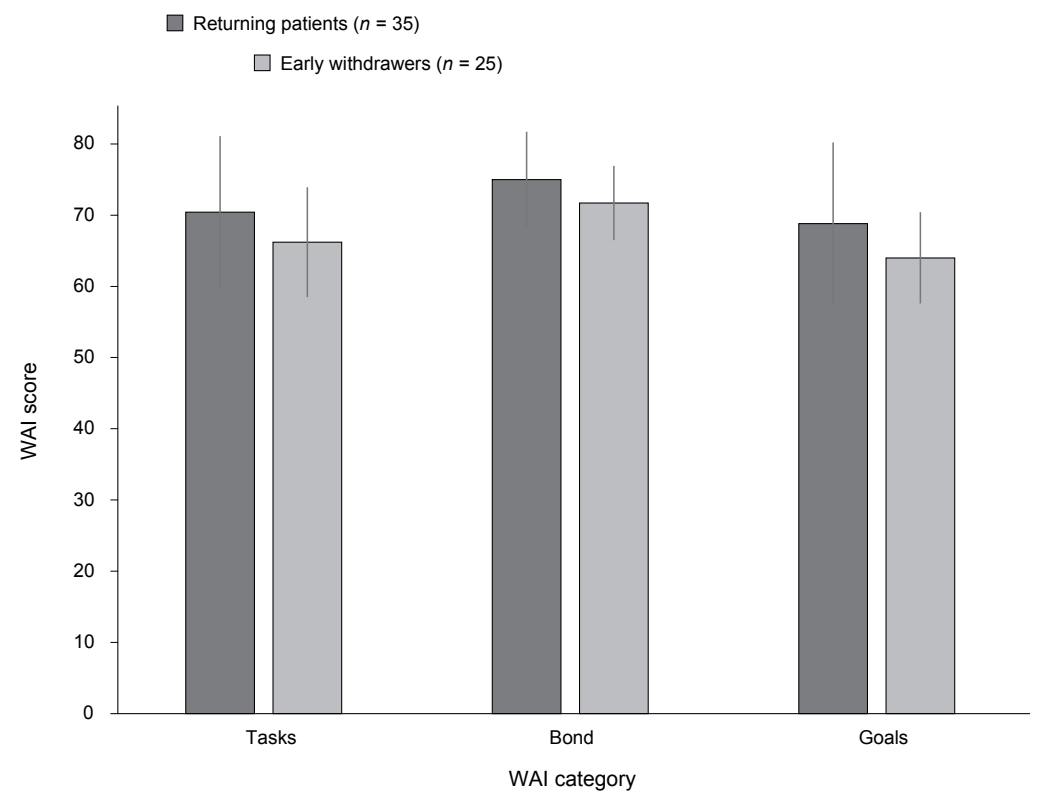

Fig. 2

Clinician scores on the Working Alliance Inventory (WAI) questionnaire, scored in categories of tasks, bond and goals (error bars indicated 1 s.d.)

\section{Satisfaction with Services questionnaire (SWS)}

The SWS is a 25-item patient questionnaire developed at Yale University in Spanish (Satisfacción con los Servicios) to evaluate patient satisfaction with clinic services and accessibility. The items are presented as yes/no responses and as five-point Likert scales (Paris et al, 2005).

\section{Clinician questionnaire}

Clinicians completed a six-question survey that recorded the therapist's gender, age, profession (e.g. psychiatrist), years active in providing therapy, treatment style/theory (e.g. cognitive therapy) and primary language. It was created for the present study to gather basic data.

\section{Statistical analysis}

Participant and clinician characteristics and WAI and SWS responses were compared between early withdrawers and participants who attended at least one additional session in the 3 observation months. Statistics were analysed with Spearman's $\rho, \chi^{2}$ and Wilcoxon tests. Statistical significance was set at $P=0.05$

\section{Results}

The overall attrition rate for the 60 participants was $42 \%$. No statistically significant differences between groups were found for patient characteristics (age, gender, occupation, income, education, diagnosis and substance misuse) or clinician characteristics (profession, age, gender, years practising, treatment style and gender match with the patient). Participants' level of education was categorised as completion of primary only for $8 \%$, secondary $46 \%$ and university $46 \%$. Clinicians had an average of 5.8 years of actively providing therapy, and treatment styles included cognitive, systemic, behavioural, interpersonal, cognitive-behavioural and gestalt therapy.

Only one of the 25 SWS questions had a statistically significant difference in response between groups: 'How would you rate the quality of services you have received?' $\left(\chi^{2}=9, P<0.05\right)$. Of the returning participants, $68 \%$ rated the quality as 'good' and 26\% 'excellent'; of the early withdrawers, 33\% rated it as 'good' and $44 \%$ 'excellent' (Fig. 1).

Participant WAI scores in all three categories (bond, goals and tasks) were not statistically different between groups. However, clinician WAI scores were statistically different between groups in all three categories (Fig. 2): tasks, $F_{1,56}=4.5$, $P<0.05$; bond, $F_{1,56}=5.7, P<0.05$; and goals, $F_{1,56}=5.7, \quad P<0.05$. There was a correlation between the participant and clinician WAI scores in the category 'tasks' for returning participants only $(\rho=0.31, P<0.05)$, but not for 'bond' or 'goals'.

\section{Discussion}

This study provides an initial understanding of factors influencing patient attrition from mental health treatment in middle- and low-income countries. Our examination of early patient 
withdrawal indicates an attrition rate of $42 \%$ after one session of therapy for this population. This figure is consistent with previous studies in highincome countries indicating an overall attrition rate of approximately $47 \%$ (Barrett et al, 2008).

The SWS responses were mostly not related to patient attrition. This suggests that satisfaction is not related to premature termination; however, we suspect that the Likert-based questions were unsuited to this population. The only significantly different response between the two groups on the SWS (Fig. 1) was in fact counter to what might have been expected: the early withdrawers rated their satisfaction 'excellent' more often than 'good', whereas the returning group rated it 'good' more often than 'excellent'.

The participants' ratings of patient-provider alliance on the WAI were also a poor indicator of premature termination; there were no differences between groups in the three categories of tasks, bond and goals. However, clinicians prospectively gave early withdrawers lower scores for alliance on tasks, bonds and goals on the WAI after only one treatment session. In addition, the scores of the returning participants were correlated with those of the clinicians in the category 'tasks', suggesting that agreement on treatment tasks may affect patient retention.

These results suggest that satisfaction and alliance questionnaires developed in high-income countries may not be effective in evaluating patient attitudes in this population, but are effective for clinician evaluation of alliance. One factor affecting their effectiveness for patients may be the Likert format of the questionnaires; in the present study this is exemplified by participants responding very positively to most questions and consequently producing an overall ceiling effect for both the SWS and the WAI data.

Another important consideration is cultural values: participants may feel inhibited from reflecting negatively on the charitable services provided by the clinic. Education level should also be considered; however, this likely had minimal impact on questionnaire comprehension, as a large majority of participants had completed at least secondary education. Nonetheless, multiple participants did comment on their unfamiliarity with the Likert-scale format. Given the present results, we feel that further examination is necessary to determine the validity of this questionnaire style for this population.

Additionally, the overall sample size should be kept in mind when interpreting the data, as well as other explanations for early termination, such as the participants' belief that an adequate level of benefit was reached in their first session.

This study does, however, suggest that clinician scores on the WAI can be used in middle- and lowincome countries, at least in rural populations, for estimates of patients' likelihood of early treatment termination. Barrett et al (2008) provide a thorough summary of effective methods of preventing patient attrition in high-income countries, particularly in relation to patient-provider alliance. The attrition rate in our study and the demonstrated predictive value of the clinician WAI for premature termination are similar to findings in high-income countries. Therefore, we believe that many of the intervention strategies used in high-income countries, as noted by Barrett et al (2008), may also be effective for patients in low- and middle-income countries.

\section{References}

Barrett, M. S., Chua, W. J., Crits-Christoph, P., et al (2008)

Early withdrawal from mental health treatment: implications for psychotherapy practice. Psychotherapy (Chicago), 45, 247-267.

Edlund, M. J., Wang, P. S., Berglund, P. A., et al (2002) Dropping out of mental health treatment: patterns and predictors among epidemiological survey respondents in the United States and Ontario. American Journal of Psychiatry, 159, 845-851.

Hansen, N. B., Lambert, M. J. \& Forman, E. M. (2002) The psychotherapy dose-response effect and its implications for treatment delivery services. Clinical Psychology: Science and Practice, 9, 329-343.

Horvath, A. O. \& Greenberg, L. S. (1989) Development and validation of the Working Alliance Inventory. Journal of Counseling Psychology, $36,223-233$.

Lhullier, A. C., Nunes, M. L. T., Antochevis, A. F., et al (2000) Change of therapist and therapy drop out in a training clinic. Aletheia, 11, 7-11.

Martin, D. J., Garske, J. P. \& Davis, M. K. (2000) Relation of the therapeutic alliance with outcome and other variables: a metaanalytical review. Journal of Counseling and Clinical Psychology, 68 438-450.

Morlino, M., Buonocore, M., Calento, A., et al (2009) First contact with psychiatric services: who leaves and who remains. General Hospital Psychiatry, 31, 367-375

Paris, M., Añez, L. M., Bedregal, L. E., et al (2005) Help seeking and satisfaction among Latinas: the roles of setting, ethnic identity, and therapeutic alliance. Journal of Community Psychiatry, 33, 299-312.

Reneses, B., Munoz, E. \& López-Ibor, J. J. (2009) Factors predicting drop-out in community mental health centres. World Psychiatry, 8, 173-177.

Rossi, A., Amaddeo, F., Sandri, M., et al (2008) What happens to patients seen only once for psychiatric services? Findings from a followup study. Psychiatry Research, 157, 53-65.

Santibáñez, P. (2003) The therapeutic alliance in psychotherapy: 'Working Alliance Inventory' in Chile. Psykhe, 12, 109-118.

Santibáñez Fernández, P. M., Román Mella, M. F. \& Vinet, E. V. (2009) Efectividad de la psicoterapia y su relación con la alianza terapéutica [Effectiveness of psychotherapy and its relationship to the therapeutic alliance]. Interdisciplinaria, 26, 267-287. 